\title{
Electronic Changes in Molybdenum Dichalcogenides on Gold Surfaces
}

\author{
Glenn G. Jernigan ${ }^{1}$, Jose J. Fonseca ${ }^{1}$, Cory D. Cress1, Mikhail Chubarov² ${ }^{2}$ Tanushree H. \\ Choudhury ${ }^{2}$, and Jeremy T. Robinson ${ }^{1}$ \\ ${ }^{1}$ Electronics Science and Technology Division, \\ US Naval Research Laboratory, Washington DC 20375 \\ 22D Crystal Consortium- Materials Innovation Platform, Materials Research Institute, \\ The Pennsylvania State University, University Park, PA 16802
}

\section{Supplemental Information:}
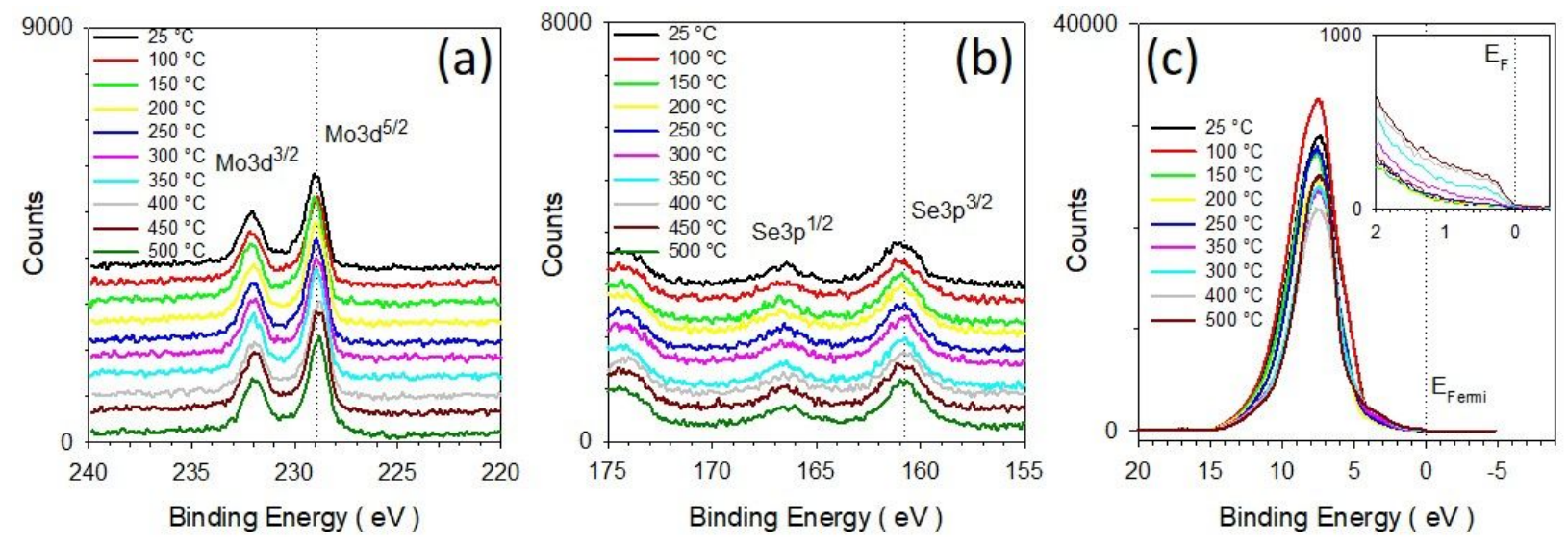

Supplement Figure S1. XPS measurements of a $\mathrm{MoSe}_{2}$ monolayer flake as annealed on Au: (a) the Mo 3d transitions and (b) the Se 3p transitions. UPS measurements (c) of a $\mathrm{MoSe}_{2}$ monolayer flake as annealed on $\mathrm{Au}$. The inset shows a blow-up of the spectra at the Fermi level. 

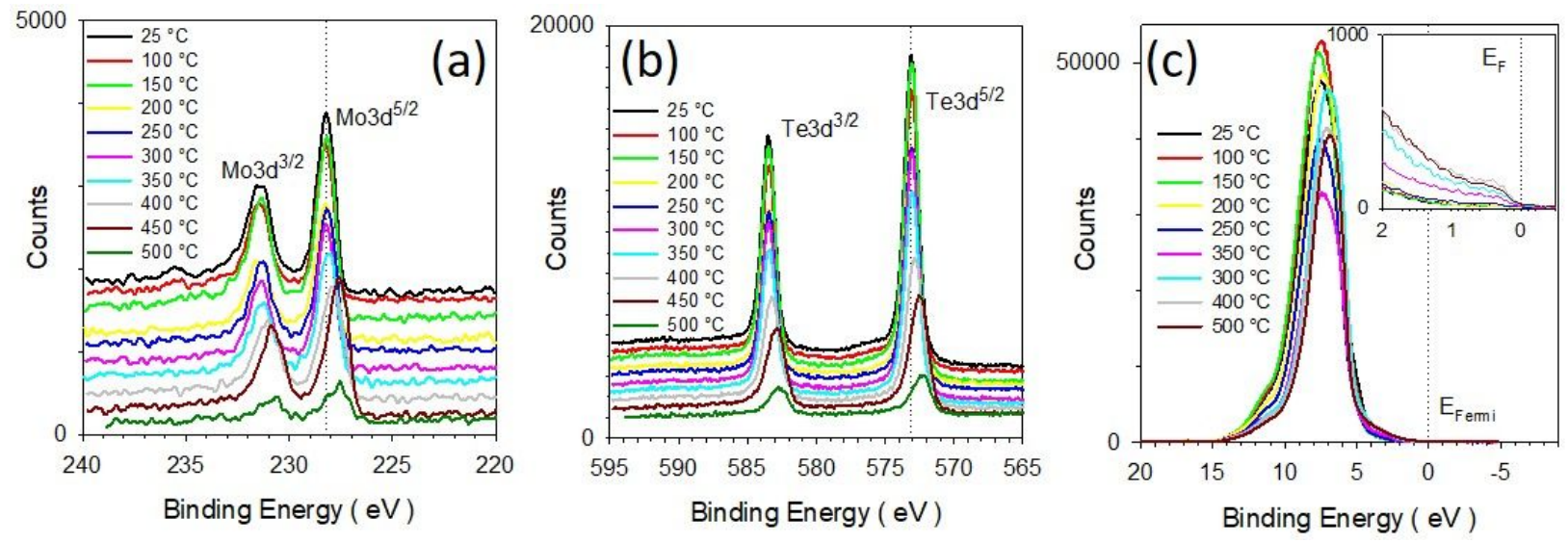

Supplement Figure S2. XPS measurements of a $\mathrm{MoTe}_{2}$ monolayer flake as annealed on $\mathrm{Au}$ : (a) the Mo $3 \mathrm{~d}$ transitions and (b) the Te $3 \mathrm{~d}$ transitions. UPS measurements (c) of a $\mathrm{MoTe}_{2}$ monolayer flake as annealed on Au. The inset shows a blow-up of the spectra at the Fermi level.
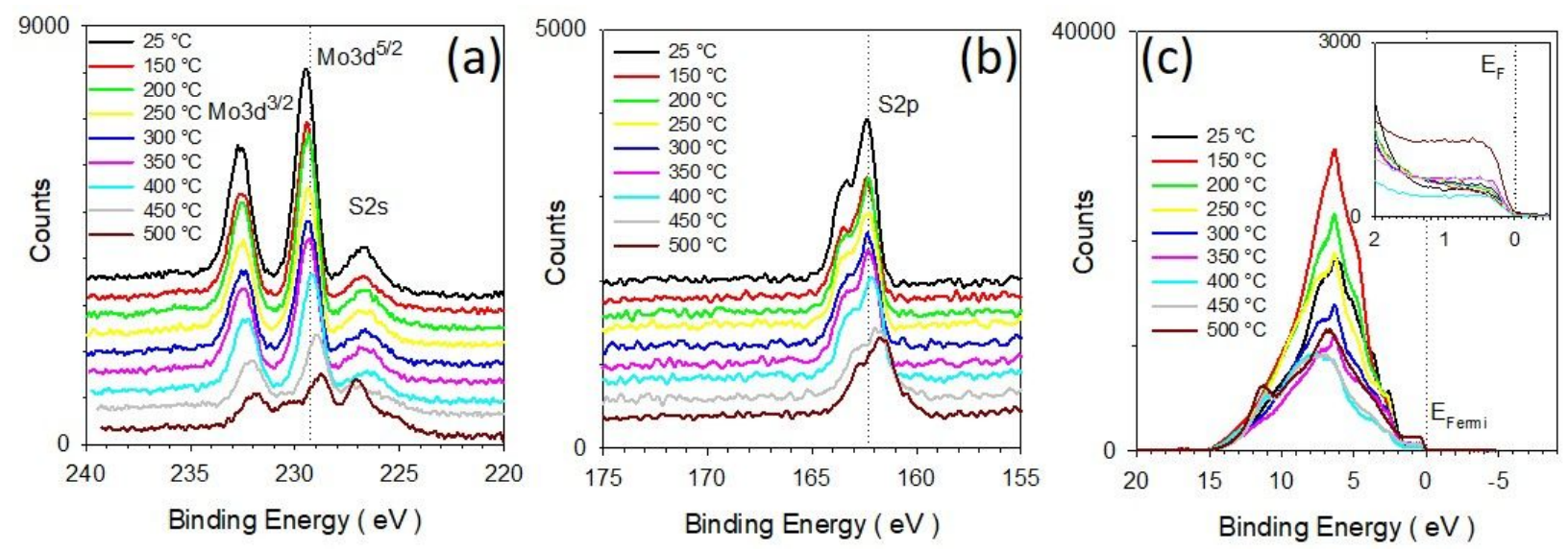

Supplemental Figure S3. XPS measurements of a helium ion $\left(\mathrm{He}^{+}\right)$damaged $\mathrm{MoS}_{2}$ monolayer flake as annealed on $\mathrm{Au}$ : (a) the Mo $3 \mathrm{~d}$ transitions and (b) the $\mathrm{S} 2 \mathrm{p}$ transitions. UPS measurements of (c) a $\mathrm{MoS}_{2}$ monolayer flake as annealed on Au. The inset shows a blow-up of the spectra at the Fermi level. 


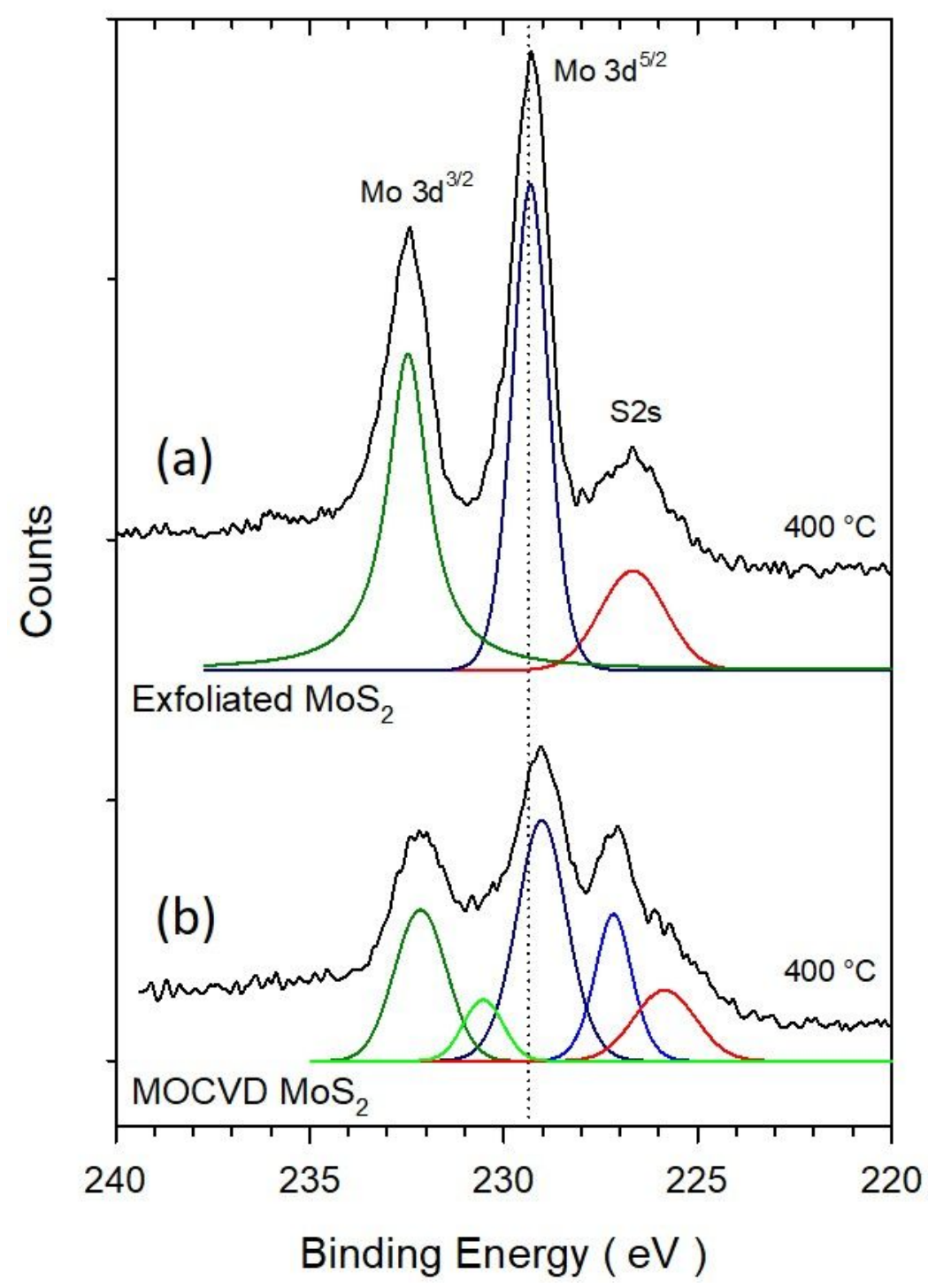

Supplemental Figure S4. Peak fitting of the XPS measurements for the Mo 3d spin splitting $\left(3 \mathrm{~d}^{5 / 2}\right.$-blue and $3 \mathrm{~d}^{3 / 2}$-green) and the S $2 \mathrm{~s}$ (red) for (a) an exfoliated $\mathrm{MoS}_{2}$ monolayer flake and (b) a MOCVD grown $\mathrm{MoS}_{2}$ film as annealed on $\mathrm{Au}$ at $400{ }^{\circ} \mathrm{C}$. The exfoliated flake is fit by a single Mo species and the MOCVD film is fit by two Mo species $\left(\mathrm{MoS}_{2}\right.$ and metallic Mo shifted to lower binding energy by $\sim 2 \mathrm{eV}$ ). The $\mathrm{S} 2 \mathrm{~s}$ broadens and shifts to lower binding energy as $\mathrm{S}$ transfers from bonding Mo atoms to bonding with the Au substrate. 


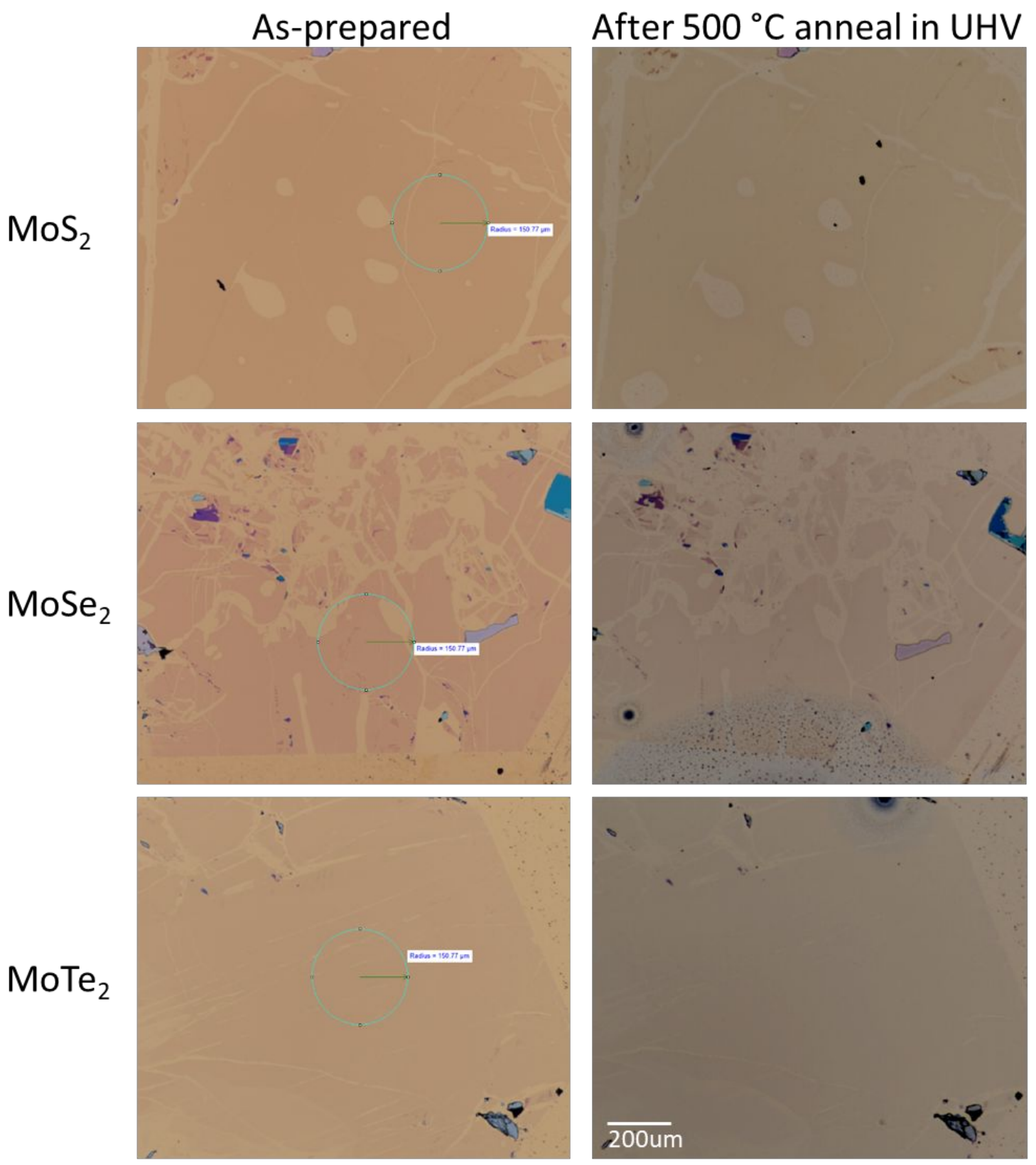

Supplemental Figure S5. Optical microscope images of $\mathrm{MoS}_{2}$ (top), $\mathrm{MoSe}_{2}$ (middle), and $\mathrm{MoTe}_{2}$ (bottom) monolayer flake samples as prepared (left side) and after XPS/UPS studies and annealing at $500{ }^{\circ} \mathrm{C}$ (right side). 

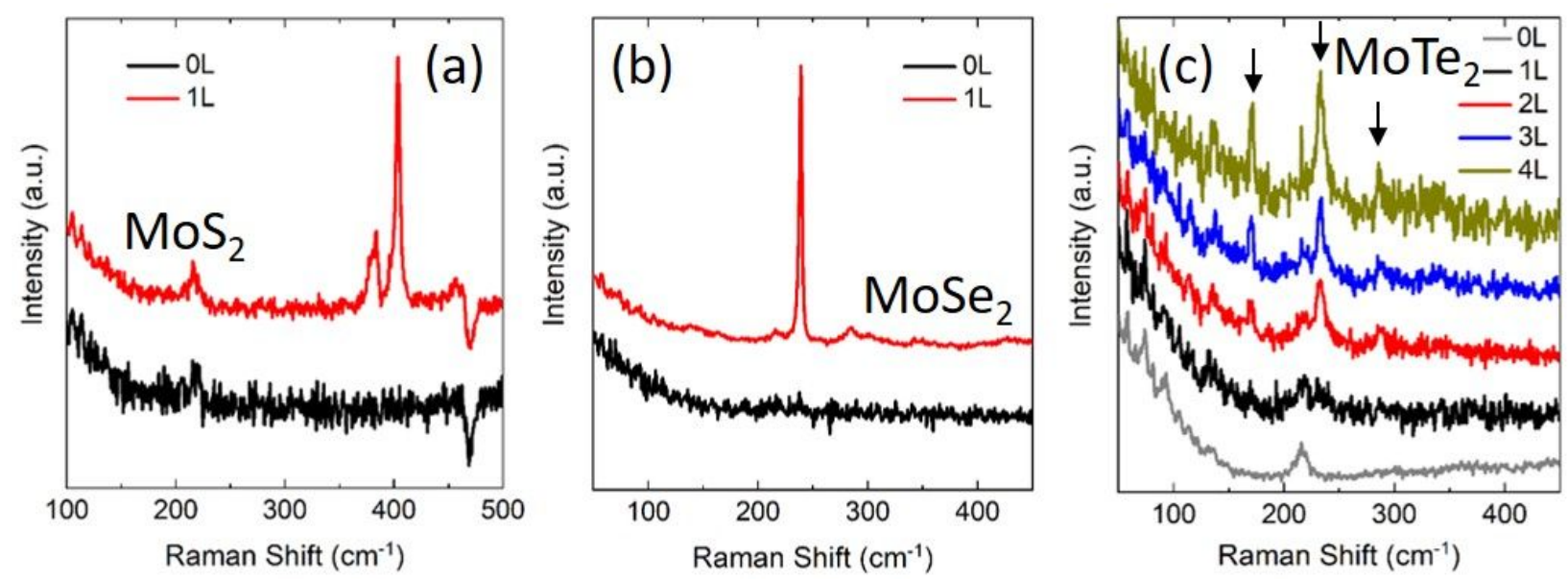

Supplemental Figure S6. Raman microscopy of single monolayer (a) $\mathrm{MoS}_{2}$, and (b) $\mathrm{MoSe}_{2}$ samples as prepared, and (c) $\mathrm{MoTe}_{2}$ multiple monolayer flake samples with a PMMA cap to prevent oxidation. 